\title{
DIRECTIONAL ASYMMETRIES OF OPTOKINETIC NYSTAGMUS: DEVELOPMENTAL CHANGES AND RELATION TO THE ACCESSORY OPTIC SYSTEM AND TO THE VESTIBULAR SYSTEM ${ }^{1}$
}

\author{
JOSH WALLMAN² AND JOSE VELEZ \\ Department of Biology, City College, City University of New York, New York, New York 10031 \\ Received January 9, 1984; Revised July 5, 1984; Accepted August 8, 1984
}

\begin{abstract}
To investigate the relation of the directional organization of the accessory optic system (AOS) to that of its principal behavioral output, optokinetic nystagmus (OKN), we measured the eye velocity during $\mathrm{OKN}$ in response to 14 directions of stimulus motion, including horizontal, vertical, cyclorotational (rotations about the optic axis), and intermediate directions in both neonatal and older chickens. We found substantial and consistent OKN asymmetries between opposite directions of stimulus motion when the stimuli were viewed monocularly; the asymmetries were largest to combinations of cyclorotational and vertical stimulus motion and to horizontal stimulus motion. The highest gain of $\mathrm{OKN}$ in the older animals was in response to two directions of stimulus motion: horizontal temporal-to-nasal and a combination of excyclorotation and downward. In addition, OKN to upward moving stimuli was consistently better than to downward stimuli. The association of high OKN gain in the older animals with the pattern of visual motion produced by head movements exciting the contralateral anterior semicircular canal suggests a possible vestibular organization of the optokinetic system.

The response pattern of the newly hatched chickens differed in three ways from that of the older animals: (1) in the non-horizontal stimulus directions the best direction was to upward and excyclorotational stimulus motion; (2) the horizontal asymmetry was somewhat less strong; and (3) the OKN gain to high velocity horizontal stimulus motion was lower. The change in directional pattern of OKN over the first weeks of life appears related to a corresponding change in anatomy of the AOS.
\end{abstract}

To see clearly during either voluntary or involuntary rotations of the head, it is necessary to produce compensatory rotations of the eyes so that the seen world remains stable. The information about angular head velocity that the brain uses to produce these eye movements evidently comes largely from the semicircular canals during head movements of high frequency and velocity and from the visual system during head movements of low frequency and velocity. The resulting eye movements are called the vestibulo-ocular reflex when only vestibular inputs are involved and are called optokinetic eye movements when only visual inputs are involved. Between these extremes there is a broad range within the spectrum of normal head movements where both visual and vestibular signals are used. For the brain to have an unambiguous head velocity signal in this critical region, it would seem necessary that the visual and vestibular signals be well matched. Since the directionally selective retinal neurons that probably provide the input to the optokinetic system in many species appear to respond to linear

\footnotetext{
${ }^{1}$ We are indebted to Joseph Su for designing and carrying out the determination of the angle of the optic axis of the eye. We are grateful to Andre Washington for performing the surgery and to Harry J. Wyatt for suggestions on this manuscript. This work was supported by $\mathrm{Na}$ tional Institutes of Health Grant EY02937 and by the Professional Staff Congress-City University of New York Research Award Program.

${ }^{2}$ To whom correspondence should be addressed.
}

motion, they would signal motion in a rectilinear, eye-centered coordinate system, whereas the canals, which respond to components of the head rotation about axes perpendicular to the canal planes, clearly signal head motion in a polar-coordinate, head-centered coordinate system.

The question therefore arises of how these disparate signals are combined. This poses no problem in the horizontal plane, since rotations of the head stimulating the horizontal canals would be associated with simple horizontal linear whole field retinal image slip, which could be added together directly (as they apparently are in the vestibular nuclei (Robinson, 1977; Waespe and Henn, 1979; Cazin et al., 1980)). The problem arises with head movements stimulating the vertical canals, since the simplest stimulus motion for the vestibular systemhead rotation stimulating only one pair of vertical canalsproduces a complicated stimulus situation for the visual system, with local movement vectors ranging from cyclorotational to linear in different parts of the visual field.

There are two plausible neural mechanisms that could resolve this question. The visual inputs signaling head movement are largely segregated from the rest of the visual system in the accessory optic system (AOS) and parts of the pretectum. Consequently, these nuclei, perhaps together with others, could transform the visual input into signals in a vestibular coordinate frame (Simpson and Hess, 1977) or into the coordinate frame of the extraocular muscles. Work on the rabbit (Simpson et al., 1979) and on the chicken (Burns and Wallman, 1981; 
McKenna and Wallman, 1981) demonstrates that different parts of the AOS are principally concerned with different directions of visual stimulation and suggests that they may, at least in part, transform the visual input into signals with directional characteristics rather like canal signals. To explore the possible close functional relationship of the vestibular system, the AOS, and the optokinetic system, we first looked for indications of what the coordinate system of the AOS might be by examining its principal behavioral output, optokinetic nystagmus (OKN). (In chickens, the AOS appears to be necessary for OKN at least to stimulus motion in directions other than horizontal (Wallman et al., 1981a, b; J. Wallman, J. Velez, and O. C. McKenna, unpublished data).) Although there is no a priori reason for expecting $\mathrm{OKN}$ measurements to reveal the underlying directional organization, we were fortunate enough to find a set of stimulus conditions in which this was the case. $\mathrm{By}$ measuring $\mathrm{OKN}$ in response to visual stimuli moving in a variety of directions, including vertical, cyclorotational (torsional), and various combinations of vertical and cyclorotational, we found that the optokinetic system is apparently not organized about a horizontal, vertical, cyclorotational coordinate frame. Rather, the strongest $\mathrm{OKN}$ is elicited by a particular combination of vertical and cyclorotational motion which approximates the pattern of visual motion that would occur on the retina during head rotations maximally exciting the anterior canals. Second, we further tested the notion that the characteristics of the AOS are manifested in OKN by measuring the pattern of directional asymmetries of the OKN of neonates and found it to be different from that of the older animals. This difference corresponds to the finding of the following paper (McKenna and Wallman, 1985) that the functional specialization of two of the nuclei of the AOS to respond to different directions of visual input has not yet occurred in newly hatched animals.

\section{Materials and Methods}

Conventions used in describing optokinetic stimuli and responses. This study seeks to describe the response of the optokinetic system to visual motion in a variety of directions and to relate it to the patterns of head movements (and thus the patterns of stimulation of the semicircular canals) with which these directions of visual motion would be associated during normal life. To aid in visualizing these relationships, consider a sphere concentric with the head of the subject. A head rotation to the right (that is, clockwise rotation about a vertical axis) without compensatory eye movements would cause the optic axis of either eye to sweep across our imaginary sphere in the same path (and produce the same visual motion) as if the head were stationary and the sphere rotated in the opposite direction (counterclockwise on a vertical axis); this head rotation would excite the right horizontal semicircular canal. Thus, we consider leftward rotation of the surrounding sphere as equivalent to a head movement exciting the right horizontal semicircular canal.

For non-horizontal head rotations the relations are the same but are more difficult to visualize, since the vertical canals are excited by rotations about horizontal axes that in most species are not aligned with the optic axes of the eyes. As above, we will describe the visual motion produced by such head movements in two ways: in terms of the direction of movement, with respect to the eye's optic axis, of the surrounding sphere of visual space (ranging from vertical to cyclorotational), and in terms of the head movements that would produce this pattern of visual motion (ranging from pitch to roll). The relationship between these two descriptions depends on the angle of the eyes in the head. Thus, for a frontal-eyed animal pitch rotation of the head produces vertical movement of the optic axis and hence vertical visual motion, whereas for a truly lateral-eyed animal (like the rabbit) this same pitch head motion produces cyclorotational visual motion.

In this study we have measured the optokinetic responses to rotations of the seen world about the vertical axis (horizontal OKN) and about a variety of horizontal axes. We will first describe these stimuli in terms of the visual motion seen along the optic axis of the right eye and then relate them to the head movements that would produce this motion. $^{3}$

Since the terms clockwise and counterclockwise denote directions in an immediately visualizable form, we will use them in preference to more formal terms like excyclorotation or extorsion. In the studies reported here only the right eye was recorded from and visually stimulated, so that clockwise cyclorotation always refers to excyclorotation.

Eye movement recording. Eye movements were recorded by the magnetic field/search coil technique. We define the magnitude of the optokinetic response as the component of the slow phase eye movement in the direction of the stimulus movement; this is, therefore, the only component measured in the studies reported here. Consequently, we arranged for the magnetic field coils to lie in vertical planes parallel to the axis of the stimulus drum rotation, with the eye coil lying in a plane perpendicular to these planes. Specifically, to record horizontal OKN the eye coil was vertical, whereas to record $\mathrm{OKN}$ in all of the directions between vertical and cyclorotational the eye coil was horizontal. To mount the coil on the eye we took advantage of the fact that in young chickens the eye is so large and the orbit so shallow that, by making a small scalp incision near the dorsal rim of the orbit, one can gain access to the sclera near the equator of the globe. By a method described and illustrated elsewhere (Wallman et al., 1982), we attached a vertical post to the sclera and to this attached either a horizontal or a vertical coil. When the coil was horizontal the direction of eye movement recorded depended only on the angle between the optic axis of the eye and the plane of the field coils; when these were perpendicular (i.e., the eye looking into the field coils), the system measured vertical eye movements; when they were parallel, it measured cyclorotational eye movements. By this method, rotations of the eye about any horizontal axis could be measured with equal ease.

Subjects, apparatus and experimental procedure. The subjects were White Leghorn chickens (Gallus gallus domesticus), hatched in the laboratory. Sixteen birds, 4 to 5 weeks old ("older birds") and 12 birds hatched within $24 \mathrm{hr}$ of the experiment ("neonates") were used.

For measurement of non-horizontal directions of $\mathrm{OKN}$, the apparatus shown in Figure 1 was used. It consisted of a cylinder, $40 \mathrm{~cm}$ in diameter and $60 \mathrm{~cm}$ long, which rotated on a horizontal axis by means of a servo-controlled variable speed motor. The inside surface of the curved part of the cylinder was lined with horizontal stripes; the endwall had radial stripes that joined the horizontal stripes, so that the optical projection of this pattern as seen from inside the cylinder approximated the longitude lines on a globe, coming together at the axis of rotation. Although we have no evidence of how optimal this pattern was for eliciting OKN, it had the advantages both of being similar to the pattern used by us and others in measuring horizontal OKN and of containing no contours that might appear to move in directions other than the actual motion.

The animal was placed in an upright acrylic container approximately a radius-distance from the end-wall of the rotating cylinder on a thin acrylic shelf extending in from outside the cylinder. Its head was restrained by closing its beak about a horizontal bar that did not appreciably intrude into the animal's visual space. To change the axis of rotation of the visual surround with respect to the animal, we kept the position of both the visual surround and the magnetic field fixed and changed the orientation of the animal within it. To permit this, the animal container was mounted onto a pivoting table on the shelf so that the pivot was under the animal's right eye; the left eye was covered with a hemispherical translucent vinyl occluder (Wallman et al., 1978).

To measure $\mathrm{OKN}$ in the various non-horizontal directions the animal container was initially set so that the optic axis of the animal's right eye was perpendicular to the axis of rotation of the cylinder, and a run

\footnotetext{
${ }^{3}$ For convenience, we will simplify the description of the visual motion by describing it in terms of the motion seen along the optic axis, although, of course, other directions of motion would be seen away from the optic axis. For example, if the optic axis sees clockwise cyclorotation, retinal cells with receptive fields to the right in visual space will view a combination of downward and clockwise cyclorotation. In this paper we will assume that the OKN to these other directions of motion will be minimized by $\mathrm{OKN}$ to opposite directions of motion on the opposite side of the optic axis (in our example, by receptive fields to the left of the optic axis viewing upward and clockwise motion).
} 

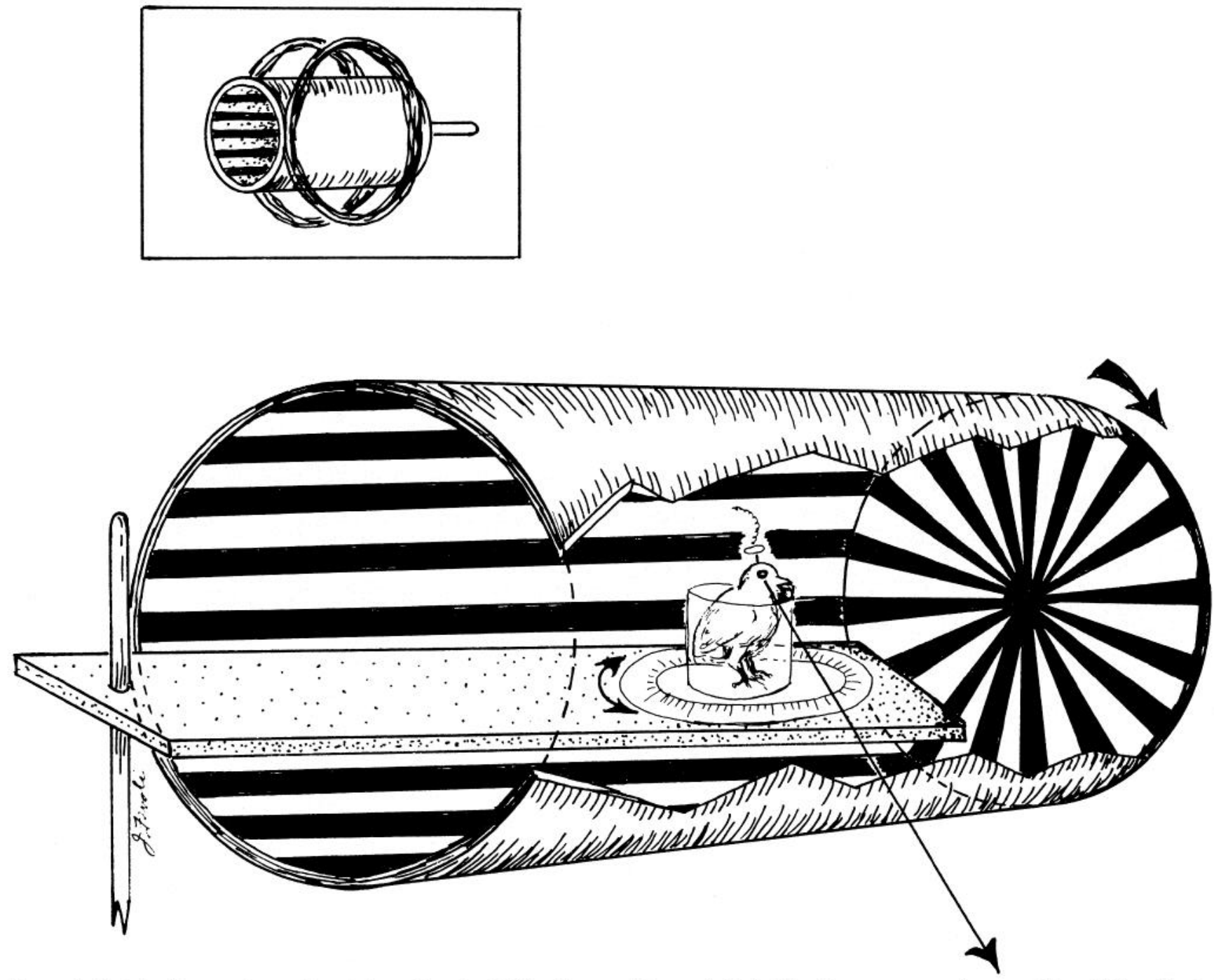

Figure 1. Sketch of apparatus and experimental animal. The drum rotates as indicated by the upper curved arrow. The relationship to the field coils is shown in the inset. The eye being recorded from is centered in the field coils and lies on the axis of rotation of the stage, which rotates as shown by the lower curved arrows. The animal shown here is positioned so that the optic axis of the right eye (straight arrow) is perpendicular to the axis of rotation of the drum. In this position (designated $0^{\circ}$ ), the bird sees downward motion. Rotating the bird to the left by $90^{\circ}$ would cause the optic axis to point toward the center of the radial pattern. In this position (designated $90^{\circ}$ ), the bird sees clockwise torsional motion.

of downward $\mathrm{OKN}$ was recorded; the animal's container was then set at different angles and other directions of OKN were recorded. Since the magnetic field was always perpendicular to the axis of stimulus rotation and the eye coil was horizontal, no change in the recording procedure was necessary, whether the eye movement response measured was purely vertical or purely cyclorotational. In this way we obtained recordings from each of the directions of visual surround rotation reported here.

In all directions, the stimulus velocity was $15^{\circ} / \mathrm{sec}$. This was chosen to be high enough that directional differences in sensitivity would be revealed ${ }^{4}$ and low enough to be within the velocity range of the neurons of the AOS (Burns and Wallman, 1981). In some experiments additional measurements were made across a range of stimulus velocities from $5^{\circ}$ to $100^{\circ} / \mathrm{sec}$.

Although the data are presented in this paper as though the visual surround always rotated clockwise and the animal was placed in 12 different positions, this, in fact, was not done, as it would have permit-

${ }^{4}$ At low velocities the gains approach 1 in all directions, presumably as a result of the large difference between the open-loop gain of the optokinetic system and the much lower closed-loop gain that is actually measured. ted the animal to see the open end of the cylinder and the supporting shelf during runs at certain positions. Instead, the animal was always oriented with its right eye looking in the $180^{\circ}$ sector away from the open end of the cylinder, and the other stimulus motion directions were obtained by reversing the direction of rotation of the cylinder.

To measure horizontal OKN we replaced the stimulus drum with a similarly driven, vertically striped vertical drum, placed the eye coil in a vertical orientation, and measured $\mathrm{OKN}$ in response to stimulus velocities from $5^{\circ}$ to $80^{\circ} / \mathrm{sec}$.

Data analysis and calibration. The signal from the eye coil went to a phase-detector and amplifier and was written out on a Gould chart recorder. This eye position signal also went to an analogue differentiator (high frequency cut-off $15 \mathrm{~Hz}$ ) to yield the eye velocity signal. The eye position channel was calibrated by attaching the eye coil to a plastic goniometer jig, located in the same position in the field as the animal's eye, and setting the coil at known angles $\left( \pm 90^{\circ}\right)$ to the field. The differentiator gain was calibrated by means of triangle-wave signals of known frequency and amplitude.

The OKN slow-phase velocity was measured from the chart records by means of a digitizing tablet interfaced to a computer. We measured the maximum slow-phase velocity within each nystagmic beat and averaged the results for the portion of a run during which the velocity was stable (minimum of $30 \mathrm{sec}$ ). Although this procedure inevitably 
yields slow-phase velocity measurements larger than those obtained by averaging over the duration of each slow phase, it is approximately equivalent to the commonly used procedure of measuring the envelope of the OKN responses as seen on recordings made at a slow chart speed (such as Fig. 2). Both procedures minimize the difficulties presented by the fact that the instantaneous eye velocity varies over the course of each nystagmic beat, particularly during $\mathrm{OKN}$ in non-horizontal directions. For each velocity point measured we also digitized the eye position at that time and used this datum to correct the velocity reading for the arc-sine response characteristic of the search coil signal. After measuring the $\mathrm{OKN}$ in each direction, the series was repeated in reverse order to reduce any sequence effects, and the two averages of slowphase velocity were averaged together. This slow-phase velocity was divided by the stimulus velocity to obtain the OKN gain.

Determination of angle of optic axis and of semicircular canals. Since we could not control where the animal looked during the $\mathrm{OKN}$ runs beyond setting the position of its head, all of our conclusions on the direction of visual motion seen in particular positions rely on our estimate of the horizontal angle of the optic axis when the eye was in the primary position.

To determine the angle of the eye's optic axis with respect to the head, the animal's head was securely held in a jig at the center of a machinist's turntable. A telescope, the barrel of which was surrounded by small lights, was aimed at the center of rotation of the turntable. An observer, looking through the telescope, blindly rotated the turntable until he judged that the corneal reflection of the telescope lights was centered in the animal's pupil and that the saccadic excursions that moved the reflection away from being centered were equally frequent to the right and to the left. This procedure was then repeated five times for each eye of each animal and averaged. Although there was a subjective element in this centering procedure, the fact that it was done blindly and the fact that the standard deviations of measurements on individual animals were invariably within $2^{\circ}$ gave us confidence in the method. Although we are actually measuring the pupillary axis by this method, we take the liberty of referring to it as the optic axis, because extensive experience in our laboratory has shown that the first corneal and both lenticular Purkinje images lie within a few degrees of the pupillary axis. The results of these measurements of the optical axes were that the two eyes were $128.4^{\circ}$ apart in the horizontal plane in older animals; that is, the optic axis of each eye was $64.2^{\circ}$ from the midsagittal plane ( $\mathrm{SD}=2.4, n=10$ ). The corresponding figure for the newly hatched animals was $64.0^{\circ}$ ( $\mathrm{SD}=2.8, n=28$ ), and for anesthetized, newly hatched animals it was $62.3^{\circ}(\mathrm{SD}=2.6, n=17)$. None of these differences is statistically significant. In practice, in the experiments reported here, we used a value of $65^{\circ}$, based on earlier results; the $1^{\circ}$ difference is within our error of alignment of the bird.

Since this work attempts to relate directions of visual motion to the vestibular system, we dissected out the anterior semicircular canals of three chickens to verify that these canals lay approximately $45^{\circ}$ from the midsagittal plane. To do this, an observer viewing the canals from above through an operating microscope set a pin parallel to what appeared to be the thin slab that best contained the canal. Another observer measured the angle between pin and beak. Although crude, this procedure indicated that the anterior canals were within $5^{\circ}$ of the expected positions.

\section{Results}

The general appearance of the OKN studied here is shown in Figure 2. The increase in the slow-phase velocity at the start of the stimulus motion and the persistence of the OKN when the lights are turned off (optokinetic afternystagmus, OKAN) were seen in all directions, although the time constants of these processes appear rather shorter $(\sim 5 \mathrm{sec}$ rise time and $3 \mathrm{sec}$ OKAN) than those reported for rabbit (Collewijn et al., 1980) or monkey (Cohen et al., 1977; Lisberger et al., 1981).

Horizontal $O K N$. The results of monocular viewing of horizontal drum rotation at a range of stimulus velocities are shown in Figure 3. A clear asymmetry is present in both older animals and neonates, as manifested by the gain being higher at all velocities for stimulus motion in the temporal-to-nasal direction. Despite considerable interindividual variability in gain, the overall shapes of the gain-velocity functions are quite similar, although there is a suggestion that $\mathrm{OKN}$ in the tem- poral-to-nasal direction of older animals falls off less rapidly at high stimulus velocities. This causes the degree of gain asymmetry between the two directions to rise rapidly above $10^{\circ} / \mathrm{sec}$, so that the ratio of gains exceeds 5 at $80^{\circ} / \mathrm{sec}$ (Fig. 3 , inset).

The OKN of newly hatched birds is similar to that of older birds in the gain-velocity characteristics, but the directional asymmetry is weaker, particularly at high stimulus velocities. This is due to both the temporal-to-nasal gain being lower and the nasal-to-temporal gain being higher than is the case in older animals. As a result, the ratio of gains in the two directions in neonales remains between 1.7 and 2.3 across all stimulus velocities tested. A curious finding in the newly hatched birds tested at low stimulus velocities was the frequent presence of brief episodes immediately following quick phases during which the eye velocity was higher than the stimulus velocity; since we measure the peak slow phase velocity within each beat, these episodes appear as gains above 1, although in fact they lasted only for fractions of a second at a time. The same phenomenon has been observed in infant rabbits (Daw and Wyatt, 1974).

The shape of the gain/velocity functions in both directions and in animals of both ages is quite similar. An indication of the degree of similarity is that all four curves are well fit by a straight line when log gain is plotted against the square root of stimulus velocity (all coefficients of determination above 0.99 ).

$O K N$ in directions other than horizontal. The OKN in nonhorizontal directions showed asymmetries as great as or greater than the horizontal OKN. The presence of these asymmetries is very consistent across individual animals (Fig. 4). Since only the direction of drum rotation differed between the two runs yielding each bar, the reality of these directional asymmetries seems unequivocal.

Since the OKN appeared normal in gross appearance in all directions, we do not think that the gain differences found could have resulted from some artifact of stimulus direction, such as the eye taking up an eccentric position during stimulation in certain directions. Although the eye did consistently deviate in the direction of the slow phases during OKN (Fig. 2 ), this deviation was greater in the directions of high $\mathrm{OKN}$ gain. Consequently, the gain asymmetries reported here could not result from decreased slow-phase velocity in those directions that caused greater eye position deviations. If anything, this effect of eye position would attenuate the asymmetries. In fact, at the stimulus velocity used in these experiments, the extent of these deviations was modest: the average difference in mean eye position between opposite directions of stimulus rotation was less than $6^{\circ}$ for all directions. This contrasts with the situation in rabbits, in which vertical stimulus motion causes the eyes to take up an extremely eccentric position in the orbit (Erickson and Barmack, 1980).

The overall directional pattern of $\mathrm{OKN}$ gain measured in this manner is shown in Figure $5 A$. To more easily visualize the directions of stimulus motion involved, one can imagine the circular surround of the figure to represent a top view of a sphere turning on a horizontal axis represented by the pivots extending above and below, such that the right side of the sphere is moving downward; an observer inside this sphere would see downward movement when looking toward the right $\left(0^{\circ}\right)$, upward to the left $\left(180^{\circ}\right)$, clockwise when looking toward the axle depicted above $\left(90^{\circ}\right)$, and so forth. (For example, the optic axis of the bird depicted in Fig. 1 is at $0^{\circ}$, corresponding to the downward stimulus motion.) The head movements of the bird that would have produced the directions of visual movement plotted here are described on the outer ring of Figure $5 A$. The data points connected by the dashed line represent the mean OKN gain of 13 older animals. The smooth curve resulted from fitting a trigonometric polynomial to the data points (see "Appendix"). It is clear that in older animals the highest OKN 


\section{stimulus motion}

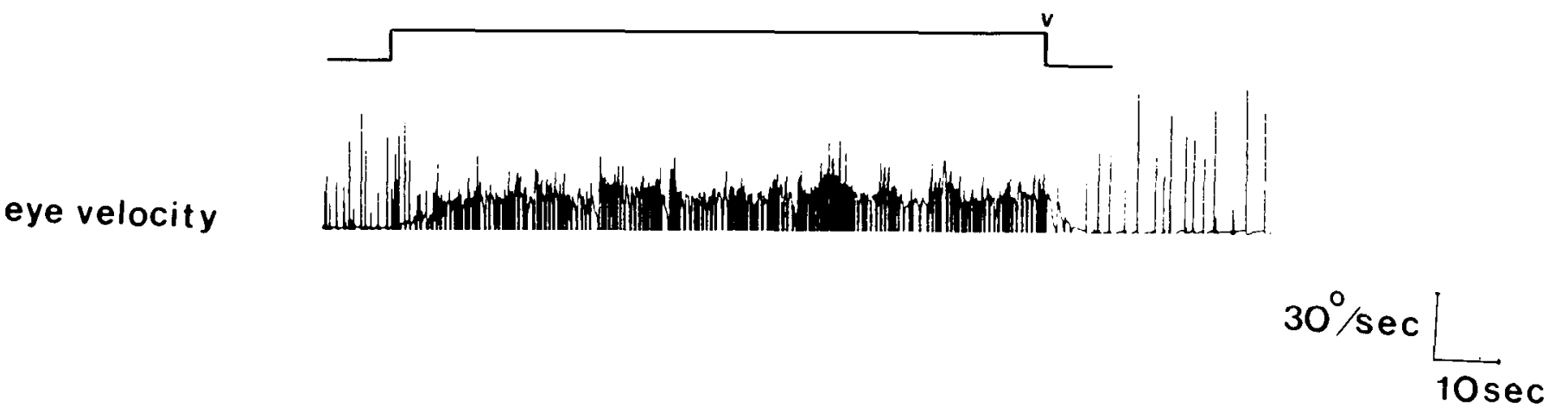

\section{eye position}

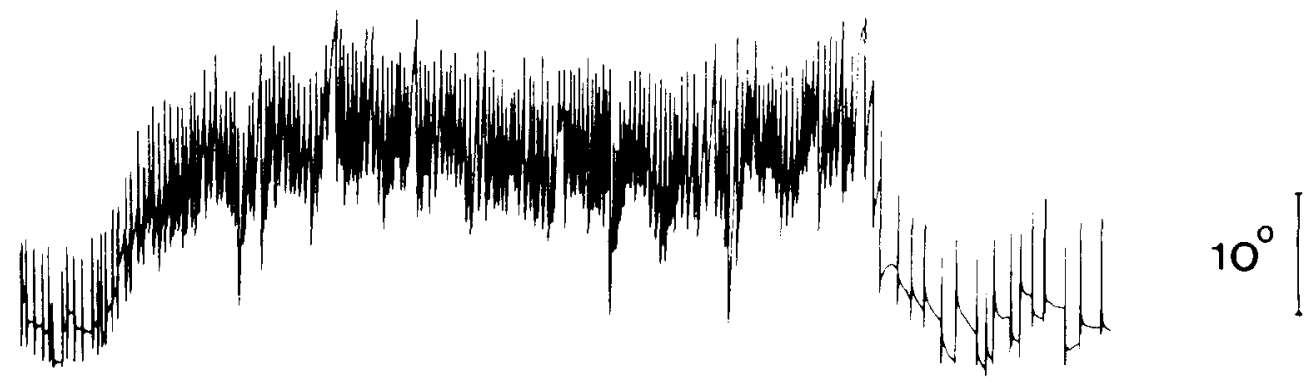

Figure 2. Examples of raw records of $\mathrm{OKN}$ in older animal. The stimulus is clockwise torsional movement presented to the right eye. Slowphase eye movements cause the eye velocity trace to move upward from the zero level present at the start and end of the trace when the stimulus drum was not moving. The downward deflections (the quick phases) are clipped at zero velocity and are not relevant to this study. Note the quick buildup of the slow-phase eye velocity after the start of the stimulus motion and its rapid decay when the lights are turned off (arrowhead). The eye position trace shows the tendency of the eyes to deviate in the direction of the stimulus motion (upward here). These records differ from those used for data analysis in that the chart was run much more slowly here and these are filtered more sharply (10- $\mathrm{Hz}$ high frequency cut-off; $48 \mathrm{~dB} /$ octave) to reduce the $25-$ to $30-\mathrm{Hz}$ uscillation that accompanies all chicken saccades and quick phases.

gain is produced to a combination of clockwise cyclorotation and downward translation. The gain to this direction is nearly as high as that of the preferred direction for horizontal OKN. A smaller peak is present at either $180^{\circ}$ (upward stimulus movement) or $160^{\circ}$ (upward with slight clockwise torsional component) in 8 of the 13 animals, although it hardly shows in Figure $5 \mathrm{~A}$ because of the smearing effect of different peak positions in different individuals. The lowest gain occurs over a broad range of directions, particularly those combining counterclockwise cyclorotation and downward translation; the gain in these directions is comparable to that of the weaker horizontal direction (nasal-to-temporal).

Comparing the gain/stimulus velocity functions of the different directions measured (Fig. 6) shows that all but three directions had functions virtually identical in shape, slope, and level. The three exceptions were $70^{\circ}, 90^{\circ}$ (clockwise torsion), and temporal-to-nasal horizontal; these stimulus directions provoked higher gain OKN across a wide range of stimulus velocities. These findings reinforce those shown in Figure $5 \mathrm{~A}$ by showing that the directional asymmetries depicted are not confined to the particular stimulus velocity used.

The results for the newly hatched birds are shown in Figure $5 B$; the highest gain in these animals occurs in response to the combination of upward translation and clockwise cyclorotation and is therefore displaced from that of the older animals. This direction corresponds rather more to pitch rotation of the head. If we rotate the curve for the neonates so that its maximum coincides with that of the older animals (Fig. 5, inset), it is clear that the curves are quite similar except that the neonates have only one peak, whereas the older animals have a second peak in a generally upward and counterclockwise direction. To assess whether the direction of peak OKN gain is significantly different between the two ages tested, we applied the same Fourier curve fitting algorithm used to generate Figure 5, $A$ and $B$, to the data of each animal separately and plotted the maxima of the resulting functions, thereby obtaining the direction of highest $\mathrm{OKN}$ gain shown in Figure $5 \mathrm{C}$. The median angle of highest $\mathrm{OKN}$ gain was $82^{\circ}$ for the older animals and $100^{\circ}$ for the newly hatched animals, a statistically significant difference (Mann-Whitney $U$ test, $p<0.005, n=9,13) .{ }^{5}$ Since there is no difference in the orientation of the optic axis between the newly hatched and older birds (see "Materials and Methods"), this difference must represent a difference in the directional asymmetries of OKN. Thus, it appears that some reorganization of the directional properties of the optokinetic system takes place during the first few postnatal weeks.

\section{Discussion}

The results presented here accomplish two things: they describe in more detail than has been done in other species the pattern of optokinetic directional asymmetries, and they dem. onstrate a developmental change in these asymmetries within the first postnatal weeks. The basic patterns of asymmetries are as follows. With respect to horizontal OKN tested monocularly, the gain at $15^{\circ} / \mathrm{sec}$ is approximately twice as high for temporal-to-nasal stimulus movement as for the opposite direction. This asymmetry is present both in older animals and in neonates; it rises rapidly with stimulus velocity in older animals but not in neonates. Such horizontal asymmetries have been reported from a large variety of species possessing laterally

\footnotetext{
${ }^{5}$ Although the data are from a circular distribution, the fact that all data points occur within $180^{\circ}$ permits use of conventional statistics rather than circular statistics.
} 


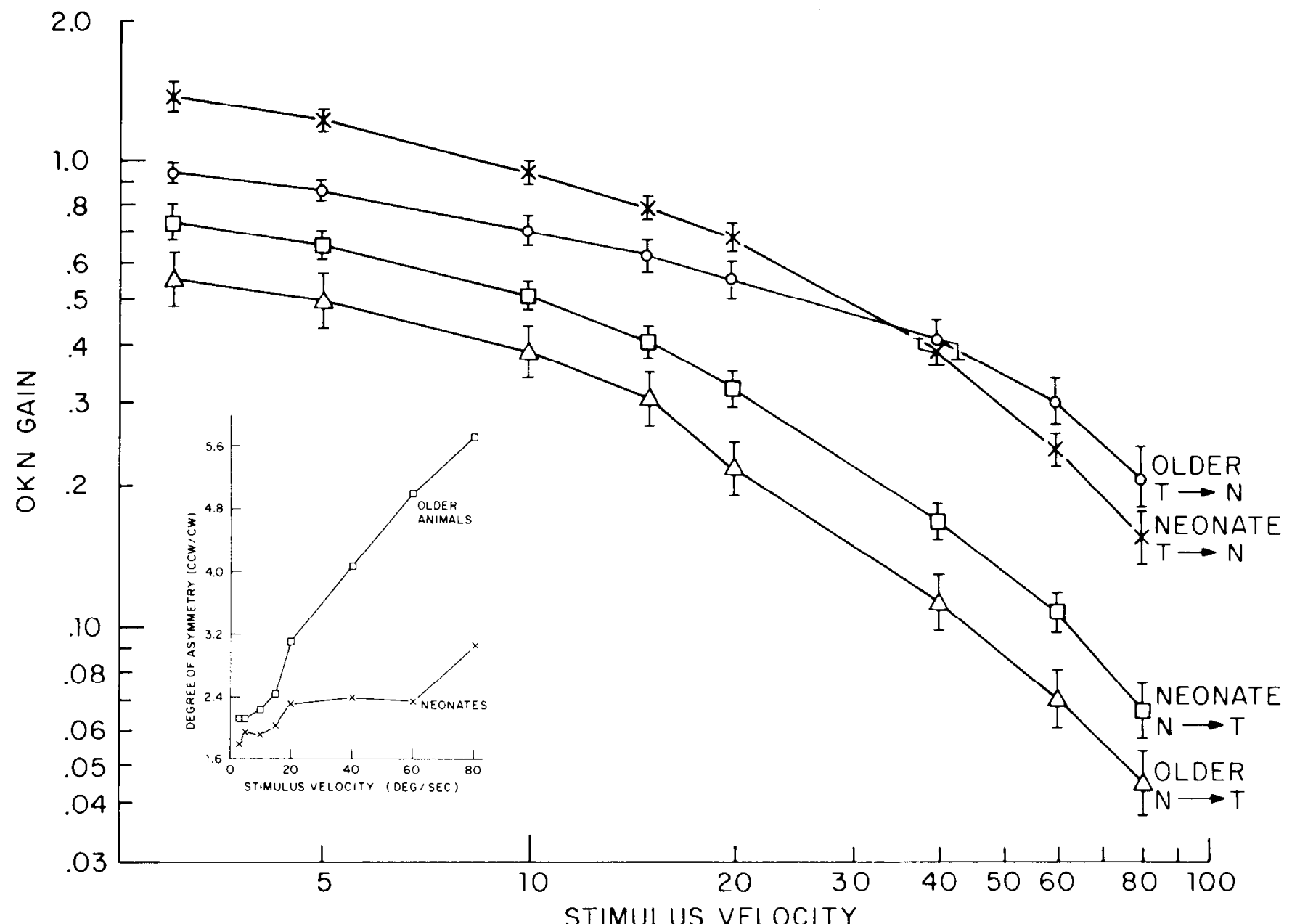

Figure 3. OKN gain to horizontal stimulus motion at a variety of velocities. The data points represent the means ( $\pm \mathrm{SE})$ of 12 older animals or of 10 younger animals. In the temporal-to-nasal direction in older animals there is a relatively higher gain at high stimulus velocities. Since the gains are plotted on a logarithmic scale, the ratio of the gains in the two directions is the vertical distance between the curves. See the text for explanation of the points at gains greater than 1 . The inset shows the average of the ratios of gains in the two horizontal directions for both older animals and neonates. Note that the inset is plotted on a linear scale.

placed eyes and nearly complete decussation of the optic tracts (rabbit: ter Braak, 1936; Collewijn, 1969; Erickson and Barmack, 1980; frog: Dieringer and Precht, 1982; goldfish: Easter, 1972; various reptiles and mammals: Tauber and Atkin, 1968; pigeon: Mowrer, 1936; Gioanni et al., 1981). With respect to non-horizontal OKN tested monocularly in the work reported here, the highest gain in older animals was to downward and clockwise stimulus cyclorotation for the right eye (Fig. 5A). This could be described in terms of eye movements as a combination of infraduction and extorsion. In most older animals there was also a second, much smaller, peak to upward motion or to upward motion with a slight clockwise cyclorotatory component. In neonates only one peak was present in the direction of upward and clockwise stimulus motion.

In other species, directional asymmetries of OKN, other than the horizontal ones mentioned above, have been adequately studied only in the vertical directions. In general, the gain to upward stimulus motion has been found to be higher than the gain to downward motion in monkeys (Takahashi and Igarashi, 1977; Matsuo et al., 1979; Matsuo and Cohen, 1984) and cats (Collins et al., 1970; Evinger and Fuchs, 1978). In rabbits, however, nearly symmetric OKN was measured to both vertical (Collewijn and Noorduin, 1972; Erickson and Barmack, 1980) and torsional (Collewijn and Noorduin, 1972) stimulation. In humans the situation is somewhat controversial, but it seems that for large stimulus patterns the gain is frequently found to be higher for upward stimulus motion as long as the stimulus velocity is not so low that the closed-loop gain is near 1 (Takahashi et al., 1978; Schor and Narayan, 1981 (Fig. 1b)). In contrast, other workers, using the electro-oculogram, have not found this asymmetry (Collins et al., 1970; Baloh et al., 1983). This disparity may be due to the effect on the electro-oculogram of the lid movements that accompany vertical eye movements, which interfere with the calibrations (Baloh et al., 1983). Some earlier reports of a lack of vertical asymmetry in human OKN (Smith, 1962; Stiefel and Smith, 1962) were based mostly on quick phase frequency, not slow phase velocity. This is a particularly unreliable indicator of OKN gain in vertical nystagmus, again because of the influence of the lids (Guedry and Benson, 1971). In the case of small stimulus patterns for which the response is probably dominated by the smooth pursuit system, the responses are approximately symmetric in both the open-loop (Dubois and Collewijn, 1979) and closed-loop (Schor and Narayan, 1981) situations. There is some evidence, however, that smooth pursuit may have its own pattern of directional asymmetries (Guedry and Benson, 1970; Baloh et al., 1983).

The interpretation of these results is affected by two technical caveats. First, although we refer the direction of stimulus motion to that seen along the optic axis, a variety of different stimulus directions is present in different parts of the visual field. Although this is of no importance for a functional descrip- 


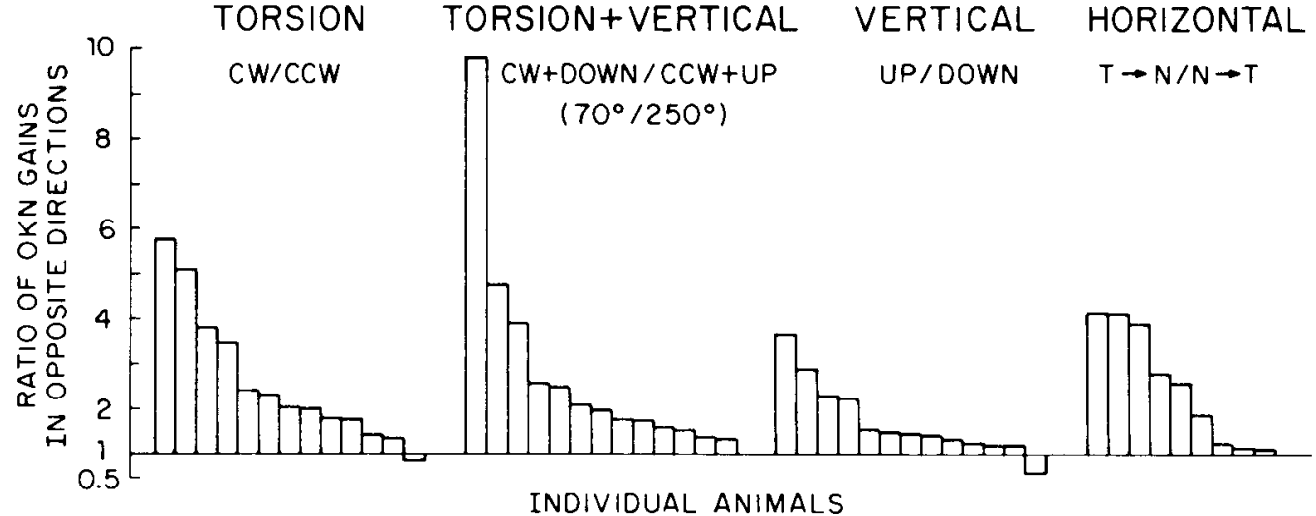

INDIVIDUAL ANIMALS
Figure 4. Variability in optokinetic gain asymmetry during stimulation of older animals in several directions at $15^{\circ} / \mathrm{sec}$. Each bar represents the ratio of one animal's response to stimulus motion in two directions differing only in whether the stimulus drum was rotating clockwise $(C W)$ or counterclockwise $(C C W)$; the bars are arranged in decreasing order of asymmetry. Note that, although the degree of asymmetry differs in the different directions and in the different individuals, the presence and direction of the asymmetries are highly reliable. $T \rightarrow N / N \rightarrow T$, temporal to nasal/nasal to temporal. tion of $\mathrm{OKN}$, since the same variety of directions is present during natural head rotations, it might be important for an understanding of the neural subunits that make up the overall optokinetic response. Thus, a particular subunit, responding to a small part of the visual field (for example, that seen by the central retina), may have different directional characteristics from that of the overall $\mathrm{OKN}$, so that the curve of $\mathrm{OKN}$ gain versus direction measured here might be different from that measured when the stimuli occupy a smaller part of the visual field. In our experiments, the OKN induced by directions seen away from the optic axis would tend to cancel out in situations in which all of the stimulus directions present in the visual field were equally potent in inducing $\mathrm{OKN}$ or in situations in which the directions seen at retinal loci equidistant from the optic axis were equally potent (as might be the case when the stimulus direction at the optic axis provokes the strongest OKN). In other situations, our method of evaluating the OKN could lead to an exaggeration of the optokinetic potency of a particular stimulus direction if a more potent direction was in the visual field at the same time. Thus, the effect of measuring the directional properties of $\mathrm{OKN}$ using a large stimulus is to broaden and to attenuate the peaks in optokinetic sensitivity (rather like the way a large slit width in a spectrophotometer broadens and attenuates spectral peaks).

The second technical problem is that we cannot, in this experimental paradigm, control where the bird chooses to look. This has the consequences that when we set the head so that the optic axis is at a particular angle, we actually include periods during which the eyes are in a small range of angles around the desired one. This effect, like the one just discussed, would be expected to broaden the peak widths measured. In both of these arguments we assume, more from parsimony than from evidence, that whatever peaks are present are symmetric ones. To the extent that this is not the case, the sources of error just discussed could lead to errors of location of the peaks.

The net effect of these technical problems is that, although we have strong evidence for the existence of these peaks, we cannot describe their shape, except to say that they are probably narrower than those shown in Figure 5, and we cannot be certain of their exact locations.

\section{OKN directional asymmetries: Relation to the vestibular system and functional significance}

We present in this paper findings that, among the nonhorizontal directions, the highest gain in the older animals $\left(82^{\circ}\right.$; heavy line in Fig. $5 \mathrm{~A}$ ) is shown to a direction of visual motion close to that produced by head movements maximally exciting the contralateral anterior semicircular canal $\left(70^{\circ}\right)$, with a smaller peak close to the direction produced by head movement exciting the ipsilateral anterior canal. Even if these directional asymmetries should prove not to be related to the canal planes, these results seem incompatible with the optokinetic system being organized in terms of a vertical component and a torsional one adding together since, although upward OKN has higher gain than downward, and clockwise has higher gain than counterclockwise, the combination of downward and clockwise yields the highest gain, as opposed to the combination of upward and clockwise that would be predicted by the addition of vertical and torsional components. Consequently, this finding requires consideration of a different scheme for the organization of the optokinetic system. Perhaps these directional coincidences between canal planes and directional peaks of OKN gain are not accidental but reflect an underlying vestibular organization of the optokinetic system. It is interesting that in the frontal-eyed species mentioned above, upward stimulus motion, which elicited stronger OKN, corresponds to excitation of both anterior canals; consequently, these results can be interpreted as compatible with our results from chickens.

The vestibular organizational scheme postulated here is made more plausible if one considers that pure optokinetic eye movements rarely occur in nature; in most circumstances the whole field retinal image slip that is the input for OKN occurs together with vestibular signals, particularly in the non-horizontal planes. Consequently, if the visual relays in the brain concerned with OKN transformed the retinal motion signals into signals in vestibular coordinates, this would facilitate adding the visual and vestibular signals. If the visual and vestibular signals were not transformed so that they shared a coordinate system, adding them together would be straightforward only in those species in which the optic axes were aligned with the canal axes; in these animals simple vertical visual movement in one of the eyes is associated with rotation in one vertical canal plane, and that in the other eye is associated with the other vertical canal plane. In all other species a simple head rotation in one canal plane is associated with a complicated combination of rotational and translational movements of the visual image.

Converting the visual motion signals into vestibular coordinates is only one plausible scheme for resolving the differences between the visual and vestibular signals. Another one would involve converting both signals into a third coordinate system, such as that of the eye muscles. Since the eye muscle coordinate system has some resemblance to the vestibular coordinate system (Graf and Simpson, 1981), such a transformation could also account for our results.

The functional significance of the directional asymmetries described here is open to question. One point of view is that these asymmetries are minimally apparent in normal life, since the directional asymmetries are less prominent at the low stimulus velocities at which the OKN gain is high (see Fig. 3 , inset, for horizontal OKN). Thus, the velocities at which the 


\section{A. OLDER ANIMALS}

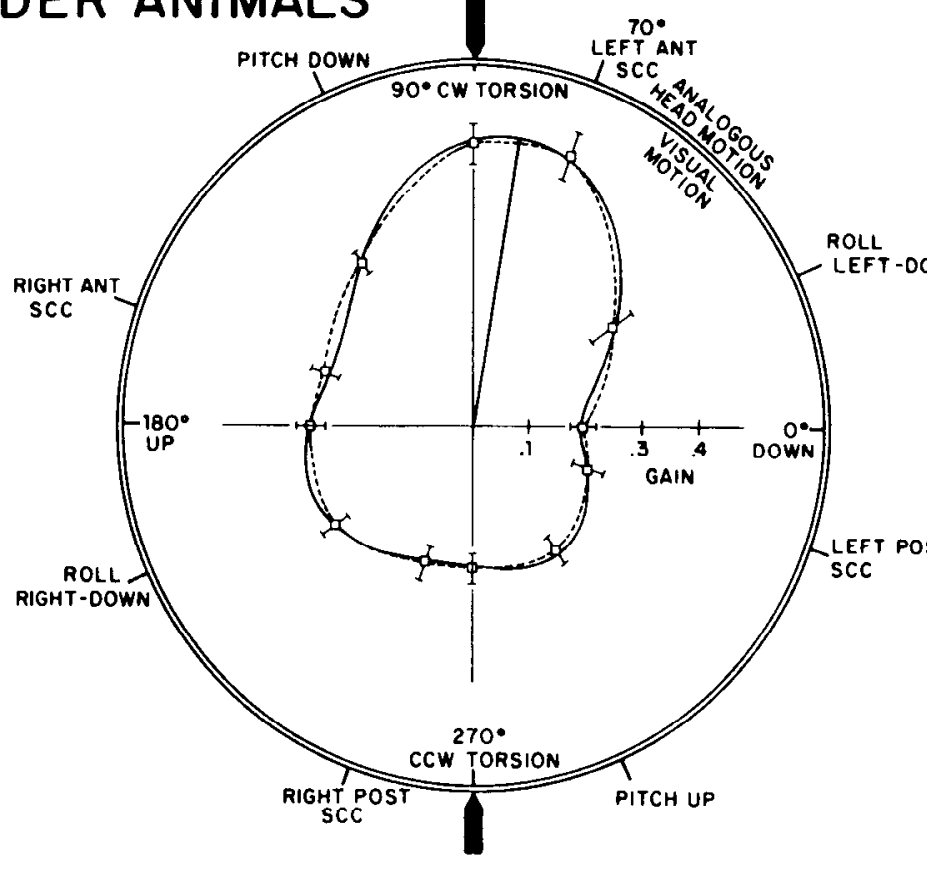

B. NEONATES

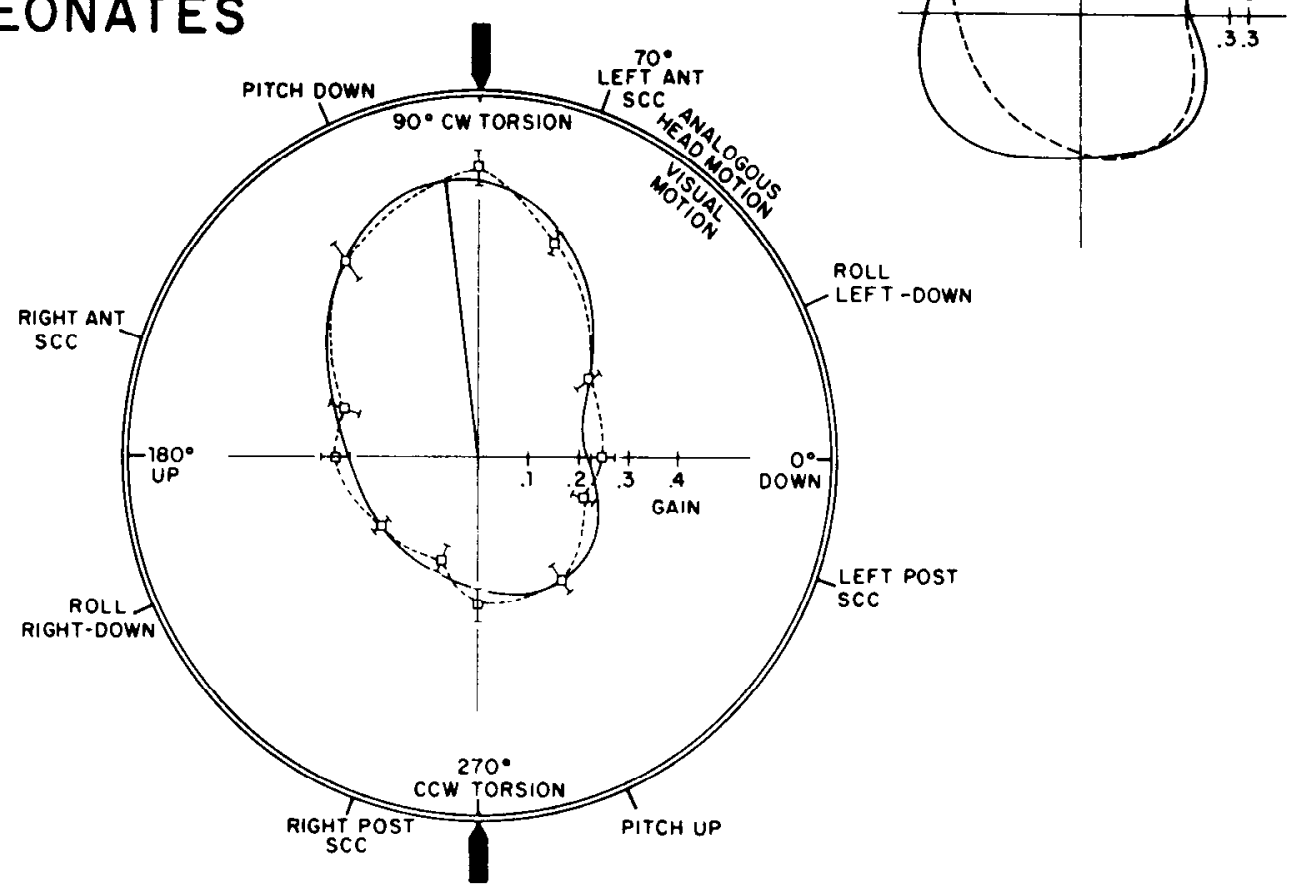

C.

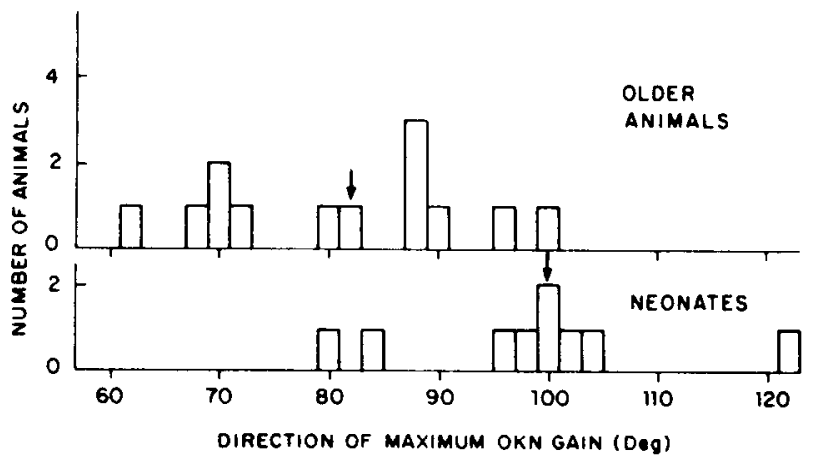

Figure 5 


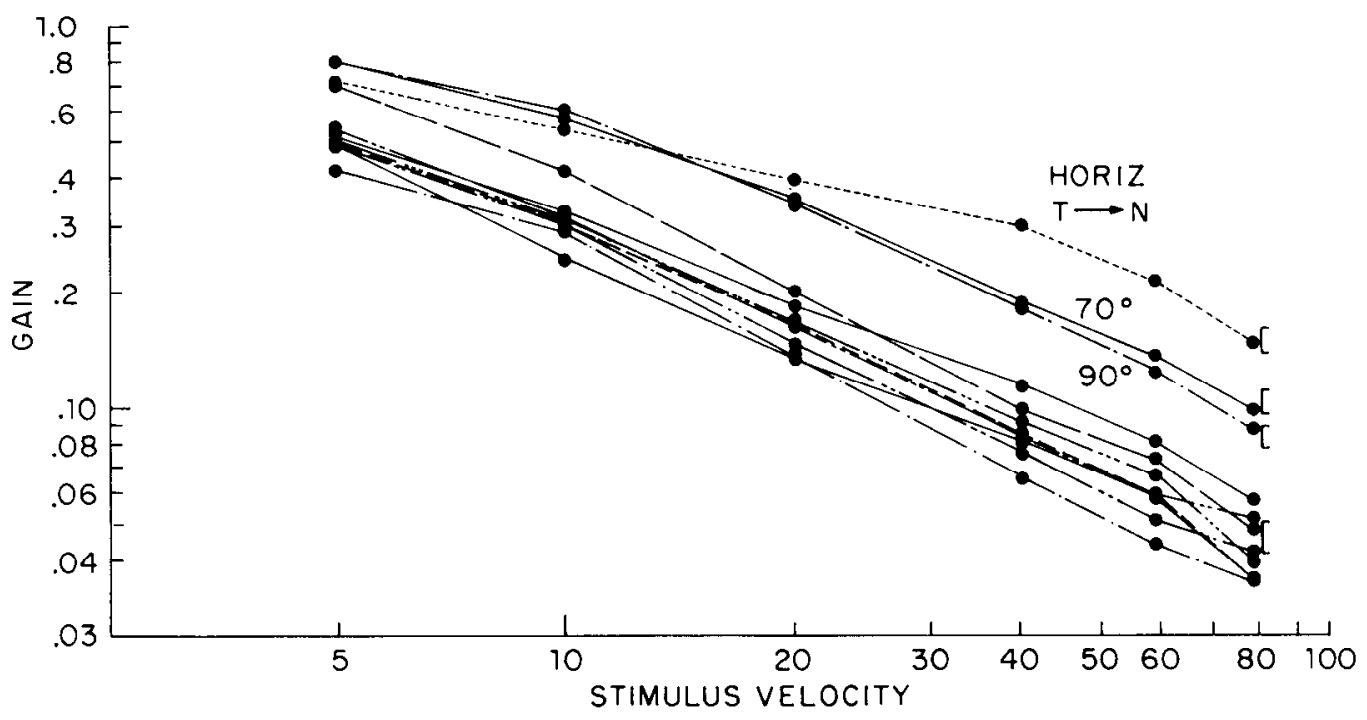

Figure 6. OKN gain-velocity functions for several directions in older animals. Note that $70^{\circ}, 90^{\circ}$, and horizontal temporal-to-nasal $($ HORIZ $T \rightarrow N$ ) provoke substantially higher gain than do the other directions $(0,160,180,250$, 270, 340, nasal-to-temporal horizontal), which are not identified in the graph. Bars to the right of the curves are average standard errors for the points on those curves. asymmetries are most manifest are those at which OKN is probably of minor importance compared to the vestibulo-ocular reflex. Furthermore, in normal life both eyes are open and the peaks of one eye's OKN gain function would tend to overlap with the nulls of the other eye's, resulting in less asymmetric OKN than that displayed in our monocular test situation. In this view, the significance of these asymmetries lies not in the behavioral output but as evidence of asymmetries of the afferent information that drives this behavior.

By contrast, it may well be that the optokinetic asymmetries do have functional significance in normal life. With respect to horizontal eye movements, a common hypothetical explanation for animals with lateral eyes having low OKN gain for nasalto-temporal stimulus motion is that it reduces the tendency for $\mathrm{OKN}$ to be provoked by forward locomotion, since it would seem counterproductive to have one's eyes tracking the passing scenery rather than being directed in front of one. With respect to non-horizontal $\mathrm{OKN}$, the asymmetries may correspond to vestibular asymmetries in the same direction, this being another example of a "systems matching" between the vestibular and visual afferents. Evidence of such corresponding vestibular and optokinetic asymmetries exists in cats (Money and Scott, 1962; Anderson, 1981; Darlot et al., 1981), in which pitch forward rotation in the dark causes a stronger vestibulo-ocular reflex than does pitch backward.

Finally, it may be inappropriate to emphasize the relation of OKN only to the semicircular canals since there is substantial evidence of an interaction of the otolith inputs with OKN. Matsuo and Cohen (1984) have shown that asymmetries of vertical $\mathrm{OKN}$ in the monkey are much stronger when the animal is placed on its side, which they interpret as suggesting that, when the monkey is in the upright posture, the otolith inputs suppress the velocity storage of OKN to upward stimulus motion.

\section{Relation of $O K N$ directional pattern to the AOS}

Since we wish to argue a relation of the patterns of OKN to the AOS and to compare our results with those of electrophysiologic work on mammals, we will briefly review the evidence for there being a general verebrate pattern of the $\Lambda O S$ contributing importantly to OKN. In those species for which substantial neurophysiological studies exist, the accessory optic neurons consistently show response characteristics suited to OKN (frogs: Katte and Hoffmann, 1980; Cochran et al., 1984; rabbits: Simpson et al., 1979; cats: Grasse and Cynader, 1982; chickens: Burns and Wallman, 1981; pigeons: Britto et al., 1981; Morgan and Frost, 1981; Rey et al., 1982). Furthermore, prominent projections from the AOS go to regions known to be involved in OKN: to the vestibulocerebellum, both directly as climbing fibers and indirectly via the inferior olive (mammals: Maekawa and Simpson, 1973; Winfield et al., 1978; Maekawa et al., 1981; birds: Brecha et al., 1980; reptiles: Reiner and Karten, 1978; fish: Finger and Karten, 1978), to the vestibular nuclei via the pons (Cazin et al., 1980), and to the oculomotor nuclei (Brecha and Karten, 1979). Finally, lesions of the AOS can cause severe impairments of vertical and torsional OKN in chickens (Wallman et al., 1981a, b), and effects have also been reported on horizontal OKN in pigeons (Gioanni et al., 1983) and chinchillas (Kimm et al., 1979) and on horizontal head nystagmus in pigeons, turtles, and frogs (Fite et al., 1979; Montgomery et al., 1982).

Not only is the AOS involved in OKN, but there is evidence in some species that it may transform visual motion from retinal to vestibular coordinates. Simpson et al. (1979) found from single-unit recordings that each nucleus of the AOS of the rabbit had a predominant direction of best response; these corresponded to excitation of the contralateral anterior canal (lateral terminal nucleus, LTN), the ipsilateral anterior canal

Figure 5. Directional patterns of OKN gain in non-horizontal directions. $A$ and $B$ show in polar coordinates the OKN gain to rotation about a variety of horizontal axes. The directions named inside the circle refer to the visual directions seen along the optic axis of the eye; those on the outside are the directions of head motion that would produce the corresponding direction of visual motion. The semicircular canal (SCC) names appear in the direction of visual motion that would be elicited by head rotation in the excitatory direction of that canal. The data points, error bars, and dashed lines represent the means and standard errors of the OKN gain measurements. The solid curved line is the best fit to these data by the first seven terms of the Fourier expansion. The heavy straight line is the direction of the maximum of this function. $A$, Older animals ( $n$ $=13) ; B$, neonates $(n=9)$. The inset shows the curve of the older animals from $A$ as a solid line, together with the curve of the neonates from $B$ rotated $18^{\circ}$ as a dashed line. The curves are scaled arbitrarily to facilitate comparison; the scaling is indicated by the hatch marks at the right for the 0.3 gain value $(O$, older animals; $N$, neonates). $C$, Frequency histogram of the direction of maximum OKN gain, as assessed by applicalion of the same Fourier curve-fitting procedure to the data of individual animals. Arrows point to medians of respective groups. The same conventions used in specifying directions in $A$ and $B$ are used here; thus, $0^{\circ}$ is downward motion and $90^{\circ}$ is clockwise cyclorotation for the right eye. The older animals are most responsive to a combination of clockwise cyclorotation and downward motion; the neonates are most responsive to clockwise cyclorotation and upward rotation. $C W$, clockwise; $C C W$, counterclockwise. 
(medial terminal nucleus, MTN), and the ipsilateral horizontal canal (dorsal terminal nucleus, DTN). Similarly, neurons in one division of the chicken AOS (the nucleus of the basal optic root pars dorsalis, nBORd) responded to pure upward movement and those in an another division (nBOR proper) responded to downward and anterior movement (Burns and Wallman, 1981). We interpreted the first as corresponding to contralateral anterior canal excitation and being analogous to the LTN, and the second as corresponding to ipsilateral anterior canal excitation and being analogous to the MTN. However, in cats, the preferred directions of neurons in the MTN do not appear to correspond, in a head-centered reference frame, to those of rabbit and chicken when the different position of the eye in the head is taken into account (Grasse and Cynader, 1982).

Thus, in both chickens and rabbits it appears that only a few stimulus directions are prominently represented in the AOS on each side of the brain and that these directions can plausibly be related to the excitatory directions of the anterior canals. The AOS may not require signals corresponding to all four vertical canals since the AOS neurons have prominent inhibitory as well as excitatory directional responses, so that responses to two orthogonal directions of rotation could resolve rotation about any horizontal axis. Although it is difficult to explain the apparent "favoring" of the anterior canals, there is other evidence of an association between the visual system and these canals. Ito et al. (1977), in a study of the vestibulo-ocular reflex elicited by electrical stimulation of individual canals, found that stimulation of the retina, optic chiasm, inferior olive, pretectum, or flocculus affected the response to simultaneous canal stimulation only in the cases of the horizontal and anterior canals; the posterior canal reflexes were unaffected.

Finally, we are not arguing that the AOS, by itself, converts the retinal directional signals into signals precisely in vestibular or eye muscle coordinates, but that some conversion rather like one of these seems to take place and that the AOS may well do part of the conversion. Furthermore, since the only function of the AOS seems related to eye movements that compensate for head movements, it would appear that fewer constraints would stand in the way of an evolutionary change in the direction of it performing such a transformation than would be the case with other parts of the visual system, which provide visual information to neural systems with several functions.

\section{Developmental changes}

Horizontal $O K N$. The principal age-related change that we find in horizontal monocular OKN is that the degree of directional asymmetry is greater in older animals, primarily because the nasal-to-temporal direction elicits weaker OKN. This result appears to be at variance with the one reported in the following paper (McKenna and Wallman, 1985), that the degree of directional asymmetry shown by 2-deoxyglucose uptake in the pretectum during $\mathrm{OKN}$ decreases with age. The present result also differs markedly from the pattern shown in frontal-eyed mammals, in which OKN of neonates is asymmetric, whereas that of adults is quite symmetric. Both of these discrepancies demand some attempt at resolution.

In mammals, progressive developmental loss of asymmetry in OKN, as well as increased high velocity sensitivity, has been attributed to the development of connectivity between the visual cortex and the pretectum in cats, monkeys, and humans (Atkinson, 1979; Malach et al., 1981; Naegele and Held, 1982). This attribution is based in part on evidence in cats showing that removal of the visual cortex (Wood et al., 1973; Montarolo et al., 1981) or manipulations known to interfere with the development of cortical binocularity (strabismus: Cynader and Harris, 1980; dark-rearing: Harris and Cynader, 1981; rearing in strobe light: Kennedy et al., 1982; monocular deprivation:
Hoffmann, 1981) also cause highly asymmetric OKN; monocular deprivation also causes a loss of ipsilateral inputs to neurons in the pretectal nucleus, the nucleus of the optic tract, on both sides of the brain (Hoffmann, 1983). It is generally assumed that the symmetric OKN of the adult is more functional in some way than the neonatal "subcortical" OKN.

In lateral-eyed animals we may presume the opposite: that the horizontal asymmetry is the more functional state, since it accompanies the lateral-eyed condition across a wide variety of taxa. Furthermore, as argued earlier, it is plausible that the asymmetry permits optokinetic responsiveness to head rotations without causing optokinetic responses during the forward translation of locomotion. We know that the visual forebrain of birds projects to the pretectum (Karten et al., 1973; Miceli et al., 1979), as well as to the AOS (Rio et al., 1983). We can speculate that, perhaps, these projections are similar to that of mammals but opposite in sign so that they increase the degree of asymmetry of the $\mathrm{OKN}$, as well as perhaps also being responsible for the enhanced high-velocity OKN responses of the older birds. According to this view, the 2-deoxyglucose labeling may reflect the activity primarily of inhibitory terminals from the forebrain. Thus, the development of inhibitory inputs to the pretectum in the older birds could account both for the decreased asymmetry of the 2-deoxyglucose results as well as the increased asymmetry of the OKN results.

Non-horizontal OKN. The OKN of newly hatched chicks and that of older animals differ in the pattern of directional asymmetries. In contrast to the older birds which had a large peak to downward and clockwise stimulation and a small peak to upward stimulation, the newly hatched birds had only one peak to upward and clockwise stimulation. Before discussing the implications of this change, we will consider whether it could be an artifact of a postnatal change in eye position. Two possibilities exist. First, if the horizontal position of the eye in the orbit moved so that the optic axis pointed about $20^{\circ}$ more nasally, this would account for the change observed, since when we aligned the bird to see clockwise and downward motion, it would in fact have been seeing clockwise and upward motion. Careful measurements, however, show that the horizontal eye position changes by less than $1^{\circ}$ (see "Materials and Methods"). Second, one could imagine the change observed being due to a torsional rotation of the eye in the orbit. The only way that a modest torsional rotation could change an upward sensitivity to a downward sensitivity is if the vertical preference was the result of a near-horizontal sensitivity, which caused the vertical bias. In this case a small eye rotation could change a generally upward sensitivity into a generally downward sensitivity. This explanation cannot account for our results since we observe a general decrease in downward sensitivity and an increase in upward sensitivity accompanying the change to increased sensitivity to clockwise torsion and down; that is to say, the peak shift we observe does not represent a change in the general up/ down sensitivity. Therefore, we are inclined to accept these data as representing a real change in the organization of the optokinetic system.

With respect to what neural changes might be involved, we have an important clue: we know something about the spatial organization of the directionally selective neurons of the AOS in both newly hatched and older chickens. Specifically, we know from single-unit recordings (Burns and Wallman, 1981) and from 2-deoxyglucose studies (McKenna and Wallman, 1981) that in older animals the units responding to upward, large field stimulus movement (which we are inclined to associate with ipsilateral anterior canal excitation) are located in nBORd, whereas units responding to downward and anteriorward stimulus movement (which we associate with contralateral anterior canal excitation) are located in the more ventral parts of the nBOR. The following paper (McKenna and Wall- 
man, 1985) shows that this directional parcellation of the nBOR, as shown by the 2-deoxyglucose method, is hardly present at all at hatching but develops rapidly within the first few postnatal weeks. Similarly, single-unit recording from newly hatched birds confirms this incompletely developed parcellation of upward- and downward-responsive units (S. Burns, unpublished data; Burns and Wallman, 1982). It seems plausible that both the anatomical and neurophysiological changes come about through a postnatal sorting out of the afferents to nBOR and that this synaptic reorganization could also account for the behavioral change reported here.

With respect to the question of what developmental processes are responsible for the change in OKN and whether they are simply maturational or experience dependent, we have only the most tentative information. Dark-reared rabbits show an enduring but modest reduction in OKN gain (Collewijn, 1977), and cats raised in unidirectional visual motion show much stronger OKN in the direction experienced (Vital-Durand and Jeannerod, 1974), although in rabbits this effect is much weaker (Daw and Wyatt, 1974). These results suggest the possible direct influence of visual experience on the development of OKN. We have found that chicks deprived of form vision for the first 3 weeks have very poor OKN in non-horizontal directions and present a 2-deoxyglucose picture rather like neonates insofar as the lack of directional parcellation of the $n B O R$ is concerned (McKenna et al., 1983). Although we know neither whether visual experience is absolutely required nor how specific this early experience must be, it is intriguing to consider the possibility that a transformation of retinal directional information into a vestibular or an oculomotor coordinate frame may be shaped by the interaction of visual and vestibular motion experience during early postnatal life.

\section{Appendix}

\section{Plotting and calculating of directional data ${ }^{6}$}

Since this paper departs from the usual conventions for dealing with directional data, we present the rationale for our method.

Directional data are commonly plotted in polar coordinates with the data points connected by straight lines. This procedure invariably distorts the shape of the function being plotted. Consider four measurements, $90^{\circ}$ apart, with an equal response in all directions. These data should be plotted as a circle but conventionally would be plotted as a square, implying that the response decreases at angles between the data points. In nearly all cases, more accurate representation is obtained if, instead of converting the data points to polar coordinates and connecting them with straight lines, one converts a series of interpolated points between the data points to polar coordinates, resulting in the lines connecting the data points being arcs, the radius of which at each point is an interpolation between the radii appropriate to the adjacent data points. This is the method used in this paper.

To compute the direction of peak response for data of this sort, the common procedure is to consider the data points as vectors and compute the mean vector. (This procedure is the one commonly used in circular statistics to compute measures of central tendency and dispersion.) Such procedures are valid only if the data are unimodal and symmetric; otherwise, the resulting mean is displaced from the peak of the distribution. This arises because the vector method actually finds the location of the center of the distribution, whereas one is usually interested in finding the location of the peak of the distribution. One could, of course, find the peak location by simply taking the direction of the largest response recorded, but this would

${ }^{6}$ By J. Wallman and M. B. Calford. be rather noisy, and one's precision would be limited by the number of directions sampled. Our procedure is to fit to the data points a function that is permitted to be asymmetric and bimodal and then take the maximum of the fitted function. The function used in this study is:

$G(\theta)=k_{0}+k_{1} \cos \theta+k_{2} \sin \theta+k_{3} \cos 2 \theta+k_{4} \sin 2 \theta+k_{5} \cos 3 \theta+k_{6} \sin 3 \theta$

where $k_{0}$ is the average OKN gain, $\theta$ is the stimulus direction expressed as an angle, and $G(\theta)$ is the calculated gain in a particular direction. This is the Fourier expansion of the data, truncated at seven terms. This function fits rather well most examples of OKN directional responses as well as visual system single-unit responses. Occasional response patterns are poorly fit by this function; these could be fit better if more terms of the Fourier expansion were used, although this would also reduce the noise-attenuating filtering qualities of the algorithm.

Algorithms of this sort work least well in the case of very broad peaks, since the maximum may be formed by a minute bump far from the center of the peak, whereas common sense would put the maximum at the center of the peak. This situation can be accommodated by seeking the two directions at which the function has fallen off to some fraction of the maximum response and using the average of these two directions as the peak response. One must be careful, in so doing, that the criterion height is not set so low as to include other peaks. This procedure was not required by the data presented here.

The authors would be glad to send a Fortran program for these computations to anyone interested in them.

\section{References}

Anderson, J. H. (1981) Behavior of the vertical canal VOR in normal and INC-lesioned cats. In Progress in Oculomotor Research, A. F. Fuchs and W. Becker, eds., pp. 395-401, Elsevier North-Holland Publishing Co., New York.

Atkinson, J. (1979) Development of optokinetic nystagmus in the human infant and monkey infant: An analogue to development in kittens. In Developmental Neurobiology of Vision, R. D. Freeman, ed., pp. 277-287, Plenum Press, New York.

Baloh, R. W., L. Richman, R. D. Yee, and V. Honrubia (1983) The dynamics of vertical eye movements in normal human subjects. Aviat. Space Environ. Med. 54: 32-38.

Brecha, N., and H. J. Karten (1979) Accessory optic projections upon oculomotor nuclei and vestibulocerebellum. Science 203: 913-916

Brecha, N., H. J. Karten, and S. P. Hunt (1980) Projections of the nucleus of the basal optic root in the pigeon: An autoradiographic and horseradish peroxidase study. J. Comp. Neurol. 189: 615-670.

Britto, L. R. G., C. L. Natal, and A. M. Marcondes (1981) The accessory optic system in pigeons: Receptive field properties of identified neurons. Brain Res. 206: 149-154.

Burns, S., and J. Wallman (1981) Relation of single unit properties to the oculomotor function of the nucleus of the basal optic root (accessory optic system) in chickens. Exp. Brain Res. 42: 171-180.

Burns, S., and J. Wallman (1982) Developmental changes in properties of single units in the avian accessory optic system. Soc. Neurosci. Abstr. 8: 204.

Cazin, L., W. Precht, and J. Lannou (1980) Pathways mediating optokinetic responses of vestibular nucleus neurons in the rat. Pfluger's Arch. 384: 19-29.

Cochran, S. L., N. Dieringer, and W. Precht (1984) Basic optokineticocular reflex pathways in the frog. J. Neurosci. 1: 13-57.

Cohen, B., V. Matsuo, and T. Raphan (1977) Quantitative analysis of the velocity characteristics of optokinetic nystagmus and optokinetic after-nystagmus. J. Physiol. (Lond.) 270: 321-344.

Collewijn, H. (1969) Optokinetic eye movements in the rabbit: Inputoutput relations. Vision Res. 9: 117-132.

Collewijn, H. (1977) Optokinetic and vestibulo-ocular reflexes in darkreared rabbits. Exp. Brain Res. 27: 287-300.

Collewijn, H., and H. Noorduin (1972) Vertical and torsional optokinetic eye movements in the rabbit. Pfluger's Arch. 332: 87-95.

Collewijn, H., B. J. Winterson, and J. van der Steen (1980) Post- 
rotatory nystagmus and optokinetic after-nystagmus in the rabbit linear rather than exponential decay. Exp. Brain Res. 40: 330-338.

Collins, W. E., D. J. Schroeder, N. Rice, R. A. Mertens, and G. Kranz (1970) Some characteristics of optokinetic eye movement patterns: A comparative study. Aerospace Med. 41: 1251-1262.

Cynader, M., and L. Harris (1980) Eye movement in strabismic cats. Nature 286: 64-65.

Darlot, C., J. Lopez-Barneo, and D. Tracy (1981) Asymmetries of vertical vestibular nystagmus in the cat. Exp. Brain Res. 41:420427.

Daw, N. W., and H. J. Wyatt (1974) Raising rabbits in a moving visual environment: An attempt to modify directional sensitivity in the retina. J. Physiol. (Lond.) 240: 309-330.

Dieringer, N., and W. Precht (1982) Compensatory head and eye movements in the frog and their contribution to stabilization of gaze. Exp. Brain Res. 47: 394-406.

Dubois, M. F. W., and H. Collewijn (1979) The optokinetic reactions of the rabbit: Relation to the visual streak. Vision Res. 19:9-17.

Easter, S. S. (1972) Pursuit eye movements in goldfish (Carassius auratus). Vision Res. 12: 673-688.

Erickson, R. G., and N. H. Barmack (1980) A comparison of the horizontal and vertical optokinetic reflexes of the rabbit. Exp. Brain Res. 40: 448-456.

Evinger, C., and A. F. Fuchs (1978) Saccadic, smooth pursuit, and optokinetic eye movements of the trained cat. J. Physiol. (Lond.) 285: 209-229.

Finger, T., and H. J. Karten (1978) The accessory optic system in teleosts. Brain Res. 153: 144-149.

Fite, K. V., A. Reiner, and S. P. Hunt (1979) Optokinetic nystagmus and the accessory optic system of pigeon and turtle. Brain Behav. Evol. 16: 192-202.

Gioanni, H., J. Rey, J. Villalobos, J. J. Bouyer, and Y. Gioanni (1981) Optokinetic nystagmus in the pigeon (Columba livia). I. Study in monocular and binocular vision. Exp. Brain Res. 44: 362-370.

Gioanni, H., J. Villalobos, J. Rey, and A. Dalbera (1983) Optokinetic nystagmus in the pigeon (Columba livia). III. Role of the nucleus ectomamillaris (nEM): Interactions in the accessory optic system. Exp. Brain Res. 50: 248-258.

Graf, W., and J. I. Simpson (1981) Relations between the semicircular canals, the optic axis, and the extraocular muscles in lateral-eyed and frontal-eyed animals. In Progress in Oculomotor Research, A. F. Fuchs and W. Becker, eds., pp. 409-417, Elsevier North-Holland Publishing Co., New York.

Grasse, K. L., and M. S. Cynader (1982) Electrophysiology of medial terminal nucleus of accessory optic system in the cat. J. Neurophysiol. 48: 490-504.

Guedry, F. E., and A. J. Benson (1970) Tracking performance during sinusoidal stimulation of the vertical and horizontal semicircular canals. In Recent Advances in Aerospace Medicine, D. E. Busby, ed., pp. 276-288, D. Reidel Publishing Co., Dordrecht, Holland.

Guedry, F. E., and A. J. Benson (1971) Nystagmus and visual performance during sinusoidal stimulation of the vertical semicircular canals. Nav. Aerospace Med. Res. Lab. Rept. 1131, pp. 1-18.

Harris, L. R., and M. Cynader (1981) The eve movements of the darkreared cat. Exp. Brain Res. 44: 41-56.

Hoffmann, K. -P. (1981) Neuronal responses related to optokinetic nystagmus in the cat's nucleus of the optic tract. In Progress in Oculomotor Research, A. F. Fuchs and W. Becker, eds., pp. 443-454, Elsevier North-Holland Publishing Co., New York.

Hoffmann, K. -P. (1983) Effects of early monocular deprivation on visual input to cat nucleus of the optic tract. Exp. Brain Res. 51. 236-246.

Ito, M., N. Nisimaru, and M. Yamamoto (1977) Specific patterns of neuronal connexions involved in the control of the rabbit's vestibuloocular reflexes by the cerebellar flocculus. J. Physiol. (Lond.) 265: 833-854.

Karten, H. J., W. Hodos, W. J. Nauta, and A. M. Revzin (1973) Neural connections of the "visual Wulst" of the avian telencephalon. Experimental studies in the pigeon (Columba livia) and owl (Speotyto cunicularia). J. Comp. Neurol. 150: 253-278.

Katte, O., and K. -P. Hoffmann (1980) Direction specific neurons in the pretectum of the frog (Rana esculenta). J. Comp. Physiol. 140: 53-57.

Kennedy, H., J. H. Courjon, and J. M. Flandrin (1982) Vestibulo-ocular reflex and optokinetic nystagmus in adult cats reared in stroboscopic illumination. Exp. Brain Kes. 48: 279-287.

Kimm, J., J. A. Winfield, and A. E. Hendrickson (1979) Visualvestibular interactions and the role of the flocculus in the vestibuloocular reflex. Prog. Brain Res. 50: 703-713.

Lisberger, S. G., F. A. Miles, L. M. Optican, and B. B. Eighmy (1981) Optokinetic response in monkey: Underlying mechanisms and their sensitivity to long-term adaptive changes in vestibuloocular reflex. J. Neurophysiol. 45: 869-890.

Maekawa, K., and J. I. Simpson (1973) Climbing fiber responses evoked in vestibulocerebellum of rabbit from visual system. J. Neurophysiol. 36: $649-666$.

Maekawa, K., T. Takeda, and M. Kimura (1981) Neural activity of nucleus reticularis tegmenti pontis - The origin of visual mossy fiber afferents to the cerebellar flocculus of rabbits. Brain Res. 210:1730.

Malach, R., N. P. Strong, and R. C. Van Sluyters (1981) Optokinetic nystagmus in long-term monocularly deprived cats. Soc. Neurosci. Abstr. 7: 733 .

Matsuo, V., and B. Cohen (1984) Vertical optokinetic nystagmus and vestibular nystagmus in the monkey: Up-down asymmetry and effects of gravity. Exp. Brain Res. 53: 197-216.

Matsuo, V., B. Cohen, T. Raphan, V. de Jong, and V. Henn (1979) Asymmetric velocity storage for upward and downward nystagmus. Brain Res. 176: 159-164.

McKenna, O., and J. Wallman (1981) Identification of avian brain regions responsive to retinal slip using 2 -deoxyglucose. Brain Res. 210: 455-460.

McKenna, O. C., and J. Wallman (1985) Functional postnatal changes in avian brain regions responsive to retinal slip: A 2-deoxy-D-glucose study. J. Neurosci. 5: 330-342.

McKenna, O. C., J. Velez, B. Taylor, and J. Wallman (1983) Visual deprivation disturbs postnatal development of the accessory optic system and optokinetic behavior. Soc. Neurosci. Abstr. 9: 820.

Miceli, D., H. Gioanni, J. Reperant, and J. Peyrichoux (1979) The avian visual Wulst. I. An anatomical study of afferent and efferent pathways. In Neural Mechanisms of Behavior in the Pigeon, A. M. Granda and J. H. Maxwell, eds., pp. 223-236, Plenum Press, New York.

Money, K. E., and J. W. Scott (1962) Functions of separate sensory receptors of nonauditory labyrinth of the cat. Am. J. Physiol. 202. $1211-1220$.

Montarolo, P. G., W. Precht, and P. Strata (1981) Functional organization of the mechanisms subserving the optokinetic nystagmus in the cat. Neuroscience 6 : 231-246.

Montgomery, N., K. V. Fite, M. Taylor, and L. Bengston (1982) Neural correlates of optokinetic nystagmus in the mesencephalon of Rana pipiens: A functional analysis. Brain Behav. Evol. 21: 137-150.

Morgan, B., and B. J. Frost (1981) Visual response characteristics of neurons in nucleus of basal optic root of pigeons. Exp. Brain Res. 42: $181-188$.

Mowrer, O. H. (1936) Maturation vs. learning in the development of vestibular and optokinetic nystagmus. J. Genet. Psychol. 48: 383 404

Naegele, J. R., and R. Held (1982) The postnatal development of monocular optokinetic nystagmus in infants. Vision Res. 22: 341 346.

Reiner, A., and H. J. Karten (1978) A bisynaptic retinocerebellar pathway in the turtle. Brain Res. 151: 163-169

Rey, J., H. Gioanni, and J. Villalobos (1982) Analyse unitaire du nucleus ectomamillaris (nEM) chez le pigeon durant le nystagmus optocinetique. C. R. Acad. Sci. Paris 295: 41-44.

Rio, J. P., J. Villalobos, D. Miceli, and J. Reperant (1983) Efferent projections of the visual Wulst upon the nucleus of the basal optic root in the pigeon. Brain Res. 271: 145-151.

Robinson, D. A. (1977) Linear addition of optokinetic and vestibular signals in the vestibular nucleus. Exp. Brain Res. 30: 447-450.

Schor, C., and V. Narayan (1981) The influence of field size upon the spatial frequency response of optokinetic nystagmus. Vision Res. 21 985-994.

Simpson, J. I., and R. Hess (1977) Complex and simple visual messages in the flocculus. In Control of Gaze by Brain Stem Neurons, R. Baker and A. Berthoz, eds., pp. 351-360, Elsevier North-Holland Publishing Co., New York.

Simpson, J. I., R. E. Soodak, and R. Hess (1979) The accessory optic 
system and its relation to the vestibulocerebellum. Prog. Brain Res. 50: 715-724.

Smith, J. L. (1962) Vertical optokinetic nystagmus. Neurology 12:48

Stiefel, J. W., and J. L. Smith (1962) Vertical optokinetic nystagmus. The normal response. Neurology 12: 245-249.

Takahashi, M., and M. Igarashi (1977) Comparison of vertical and horizontal optokinetic nystagmus in the squirrel monkey. ORL 39: 321-329.

Takahashi, M., S. Sakurai, and J. Kanzaki (1978) Horizontal and vertical optokinetic nystagmus in man. ORL 40: 43-52.

Tauber, E. S., and A. Atkin (1968) Optomotor responses to monocular stimulation: Relation to visual system organization. Science 160: 1365-1367.

ter Braak, J. W. G. (1936) Research on optokinetic nystagmus. Dutch Arch. Human Anim. Physiol. 21: 309-375.

Vital-Durand, F., and M. Jeannerod (1974) Role of visual experience in the development of optokinetic response in kittens. Exp. Brain Res. 20: 297-302.

Waespe, W., and V. Henn (1979) The velocity response of vestibular nucleus neurons during vestibular, visual, and combined angular acceleration. Fxp. Rrain Res. 37: 337-347
Wallman, J., C. Ledoux, and M. B. Friedman (1978) Simple devices for restricting the visual fields of birds. Behav. Res. Methods Instrument. 10: 401-403.

Wallman, J., O. C. McKenna, S. Burns, J. Velez, and B. Weinstein (1981a) Relation of accessory optic system and pretectum to optokinetic responses in chickens. In Progress in Oculomotor Research, A. Fuchs and W. Becker, eds., pp. 435-442, Elsevier North-Holland Publishing Co., New York.

Wallman, J., J. Velez, and O. C. McKenna (1981b) Lesions in avian accessory optic system severely disrupt optokinetic nystagmus in non-horizontal directions. Soc. Neurosci. Abstr. 7: 299.

Wallman, J., J. Velez, B. Weinstein, and A. E. Green (1982) Avian vestibuloocular reflex: Adaptive plasticity and developmental changes. J. Neurophysiol. 48: 952-967.

Winfield, J. A., A. Hendrickson, and J. Kimm (1978) Anatomical evidence that the medial terminal nucleus of the accessory optic tract in mammals provides a visual mossy fiber input to the flocculus. Brain Res. 151: 175-182

Wood, C. C., P. D. Spear, and J. J. Braun (1973) Direction-specific deficits in horizontal optokinetic nystagmus following removal of visual cortex in the cat. Brain Res. 60: 231-237. 